\title{
THE METHOD OF ESTIMATING LIFETIMES OF AIRCRAFT DEVICES OPERATING UNDER AGEING-ATTRIBUTABLE WEARING CONDITIONS
}

\author{
MARIUSZ WAŻNY \\ Military University of Technology, Warsaw, Poland \\ e-mail: mariusz.wazny@wat.edu.pl
}

\begin{abstract}
The paper presents a probabilistic method of assessing lifetimes of selected structural components or assemblies of devices/systems affected by destructive processes that occur during aircraft operation. Reliability status of the device is evaluated by means of diagnostic or operational parameters. It is assumed that these devices (systems, assemblies) operate reliably if effects of wear and tear processes described by diagnostic parameters do not exceed boundary conditions/regimes. From the mathematical aspect, the method has been based on difference equations from which, when rearranged, a partial differential equation of the Fokker-Planck type is derived. A density function of the component wearing is a particular solution to this equation. With the density function of the component wearing applied, after suitable rearrangements, one can determine a density function of time for the exceeding the boundary condition. Now, with the density function of time of reaching the boundary condition found, and after rearrangement of this function, one receives dependences that can be applied to determine lifetime of the device given consideration. An example at the end of the paper illustrates how this method can be applied to analyse an airborne sighting system.
\end{abstract}

Keywords: reliability, lifetime, destructive processes, permissible condition

\section{Introduction}

Any manifestations of aeronautical engineering, i.e. aeronautical devices, systems, etc., make design engineers, manufacturers and users meet requirements connected with maintaining high values of safety and reliability parameters. Examining the safety and reliability of an aircraft throughout the operational process involves predictions about health/maintenance status of particular devices and systems of an aircraft and the aircraft itself as a platform combining all the above-mentioned elements. Analyzing an aircraft as an object intended to provide, e.g. transportation of passengers and cargo, we can assume that the operating conditions are of special importance compared with other popular means of transport (Pamuła, 2011). A series of factors make values of parameters that describe health/maintenance status of an aircraft change over time. Destructive processes resulting from overloads, friction, vibration, ageing processes, etc. prove to have the crucial effect on that change.

An aircraft is a platform upon which systems like the following ones are integrated:

- automatic control systems,

- communication systems,

- avionic systems,

- air armament systems,

- sighting systems,

- systems to abandon the aircraft in emergency, etc. 
The health/maintenance status of aircraft devices is mainly evaluated with a set of diagnostic parameters. The effect of destructive processes is visible in changes of values of diagnostic parameters, which cause increments in deviations from nominal values of these parameters. Deviations from nominal values of diagnostic parameters are used to estimate reliability of a given device. This question is addressed in (Niu et al., 2011; Tomaszek and Wróblewski, 2001; Tomaszek et al., 2004, 2011, 2013; Tomaszek and Szczepanik, 2005; Ye et al., 2011; Zhanshan and Krings, 2008). Among the above mentioned works, (Niu et al., 2011) is an interesting publication where health/maintenance status of a piece of equipment is analysed with the Mahalanobis distance indicator and the Weibull distribution applied.

This paper is an attempt to analyse and describe degradation of health/maintenance status of selected devices as a result of destructive processes affecting them.

Classification of correlations between effects of destructive processes and changes in values of diagnostic parameters is presented by Ważny (2011a). The density function of changes in deviations of the diagnostic parameter has been determined in this article, with the following assumptions applied:

- the device health is determined by one dominant diagnostic parameter (its current value is denoted by $x$ ),

- the change in value of the diagnostic parameter due to the destructive effect of ageing processes occurs as the calendar time passes by,

- the deviation of the diagnostic parameter from the nominal value is

$$
z=\left|x_{p}-x_{n}\right|
$$

where $x_{p}$ is measured value of the diagnostic parameter, $x_{n}$ - nominal value of the diagnostic parameter,

- the value of the diagnostic parameter deviation determines the level of reliability of a given structural component. If it remains within the interval $z \in\left[0, Z_{d}\right]$, the component will be recognised as serviceable (fit for use). Otherwise, it will be recognised unserviceable (unfit for use),

- the increase in the diagnostic parameter deviation against the calendar time satisfies the relationship

$$
\frac{d z}{d t}=c
$$

where $c$ is a random variable that depends on ageing processes, $t$ - the calendar-based time.

\section{Determining the density function of changes in values of deviations of the diagnostic parameter}

It is assumed that the intensity of growth of the component wear and tear takes the same form as the failure rate for the Weibull distribution

$$
\lambda(t)=\frac{\alpha}{\theta} t^{\alpha-1}
$$

where $\alpha$ and $\theta$ are constants in the Weibull distribution with the following denotations: $\alpha$ - the shape factor, $\theta$ - the scale factor.

The stochastically approached dynamics of changes in values of diagnostic parameters, including the parameter deviation, is described by difference equation. Let $U_{z, t}$ denote the probability that at the time instance $t$ the diagnostic parameter deviation takes value $z$. 
A difference equation takes the following form for the assumed conditions

$$
U_{z, t+\Delta t}=\left(1-\frac{\alpha}{\theta} t^{\alpha-1} \Delta t\right) U_{z, t}+\frac{\alpha}{\theta} t^{\alpha-1} \Delta t U_{z-\Delta z, t}
$$

where $\Delta z$ is the increment in the diagnostic parameter deviation in the time interval $\Delta t$.

Equation (2.2) written down in functional notation takes the following form

$$
u(z, t+\Delta t)=\left(1-\frac{\alpha}{\theta} t^{\alpha-1} \Delta t\right) u(z, t)+\frac{\alpha}{\theta} t^{\alpha-1} \Delta t u(z-\Delta z, t)
$$

where $u(z, t)$ is the density function of the diagnostic parameter deviation, $\left[1-(\alpha / \theta) t^{\alpha-1} \Delta t\right]-$ probability that in the time interval $\Delta t$ there will be no increment in the diagnostic parameter deviation, $(\alpha / \theta) t^{\alpha-1} \Delta t$ - probability that in the time interval $\Delta t$ there will be the $\Delta z$ increment in the parameter deviation, and the following condition is satisfied

$$
\frac{\alpha}{\theta} t^{\alpha-1} \Delta t \leqslant 1
$$

Rearrangement of equation (2.3) into a partial differential equation results in the following approximation

$$
\begin{aligned}
& u(z, t+\Delta t)=u(z, t)+\frac{\partial u(z, t)}{\partial t} \Delta t \\
& u(z-\Delta z, t)=u(z, t)-\frac{\partial u(z, t)}{\partial z} \Delta z+\frac{1}{2} \frac{\partial^{2} u(z, t)}{\partial z^{2}}(\Delta z)^{2}
\end{aligned}
$$

Having substituted relationships (2.4) into equation (2.3) and with some rearrangements done, the following is arrived at

$$
\frac{\partial u(z, t)}{\partial z}=-\frac{\alpha}{\theta} t^{\alpha-1} \Delta z \frac{\partial u(z, t)}{\partial z}+\frac{1}{2} \frac{\alpha}{\theta} t^{\alpha-1}(\Delta z)^{2} \frac{\partial^{2} u(z, t)}{\partial z^{2}}
$$

Consideration is given to the increment in the diagnostic parameter deviation per time unit (when $\Delta t=1$ ), hence

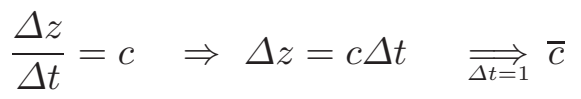

where $\bar{c}$ denotes increment in the diagnostic parameter deviation per one time unit.

The final form of equation (2.5) is as follows

$$
\frac{\partial u(z, t)}{\partial z}=-\underbrace{\frac{\alpha \bar{c}}{\theta} t^{\alpha-1}}_{\gamma(t)} \frac{\partial u(z, t)}{\partial z}+\frac{1}{2} \underbrace{\frac{\alpha \bar{c}^{2}}{\theta} t^{\alpha-1}}_{\beta(t)} \frac{\partial^{2} u(z, t)}{\partial z^{2}}
$$

The solution to equation (2.6) takes the form

$$
u(z, t)=\frac{1}{\sqrt{2 \pi A(t)}} \exp \left(-\frac{(z-B(t))^{2}}{2 A(t)}\right)
$$

where $B(t)$ is an average value of the diagnostic parameter deviation for the operating time $t$

$$
B(t)=\int_{0}^{t} \gamma(t) d t
$$


$A(t)$ - a variance of the diagnostic parameter deviation for the operating time $t$

$$
A(t)=\int_{0}^{t} \beta(t) d t
$$

With integrals (2.8) and (2.9) calculated, the following expressions are arrived at

$$
B(t)=\int_{0}^{t} \frac{\alpha \bar{c}}{\theta} t^{\alpha-1} d t=\frac{\bar{c}}{\theta} t^{\alpha} \quad A(t)=\int_{0}^{t} \frac{\alpha \bar{c}^{2}}{\theta} t^{\alpha-1}=\frac{\bar{c}^{2}}{\theta} t^{\alpha}
$$

Hence, relationship (2.7) takes the following form

$$
u(z, t)=\frac{1}{\sqrt{2 \pi \frac{\bar{c}^{2}}{\theta} t^{\alpha}}} \exp \left(-\frac{\left(z-\frac{\bar{c}}{\theta} t^{\alpha}\right)^{2}}{2 \frac{\bar{c}^{2}}{\theta} t^{\alpha}}\right)
$$

Relationship (2.11) presents the density function of the diagnostic parameter deviation from the nominal value.

With the following substitutions

$$
\frac{\bar{c}}{\theta}=b \quad \frac{\bar{c}^{2}}{\theta}=a
$$

relationship (2.11) takes the following form

$$
u(z, t)=\frac{1}{\sqrt{2 \pi a t^{\alpha}}} \exp \left(-\frac{\left(z-b t^{\alpha}\right)^{2}}{2 a t^{\alpha}}\right)
$$

With the density function found, one can write down a relationship for reliability with respect to time of the diagnostic parameter deviation increasing up to the boundary value. The formula takes the form

$$
R(t)=\int_{-\infty}^{z_{d}} u(z, t) d z
$$

where $z_{d}$ is a permissible value of the diagnostic parameter deviation.

\section{Determining distribution of time of exceeding the permissible condition by the diagnostic parameter deviation}

The probability of exceeding the boundary value by the diagnostic parameter with the density function of changes in the diagnostic parameter deviation can be written down in the following form

$$
Q\left(t, z_{d}\right)=\int_{z_{d}}^{\infty} \frac{1}{\sqrt{2 \pi a t^{\alpha}}} \exp \left(-\frac{\left(z-b t^{\alpha}\right)^{2}}{2 a t^{\alpha}}\right) d z
$$

The density function of the distribution of time of exceeding the permissible value of the diagnostic parameter $z_{d}$ equals

$$
f(t)=\frac{\partial}{\partial t} Q\left(t, z_{d}\right)
$$


With (3.1) taken into account, this equation takes the form

$$
f(t)=\frac{\partial}{\partial t} \int_{z_{d}}^{\infty} \frac{1}{\sqrt{2 \pi a t^{\alpha}}} \exp \left(-\frac{\left(z-b t^{\alpha}\right)^{2}}{2 a t^{\alpha}}\right) d z
$$

Now, the time derivative of the integrand for relationship (3.3) can be found

$$
f(t)=\int_{z_{d}}^{\infty}\left[\frac{2\left(z-b t^{\alpha}\right) b \alpha t^{\alpha}+\left(z-b t^{\alpha}\right)^{2} \alpha}{2 a t^{\alpha+1}}-\frac{\alpha}{2 t}\right] u(z, t) d z
$$

In order to calculate integral (3.4), we need to determine an antiderivative. We assume the following form of the antiderivative of the integrand in relationship (3.4)

$$
w(z, t)=u(z, t) \theta(z, t)
$$

The derivative of the indefinite integral with respect to the variable $z$ is equal to the integrand of relationship (3.4). Hence

$$
\frac{\partial u(z, t)}{\partial z} \theta(z, t)+u(z, t) \frac{\partial \theta(z, t)}{\partial z}=\left[\frac{2\left(z-b t^{\alpha}\right) b \alpha t^{\alpha}+\left(z-b t^{\alpha}\right)^{2} \alpha}{2 a t^{\alpha+1}}-\frac{\alpha}{2 t}\right] u(z, t)
$$

Now, the derivative $\partial u(z, t) / \partial z$ is calculated

$$
\frac{\partial u(z, t)}{\partial z}=\frac{1}{\sqrt{2 \pi a t^{\alpha}}} \exp \left(-\frac{\left(z-b t^{\alpha}\right)^{2}}{2 a t^{\alpha}}\right)\left[-\frac{2\left(z-b t^{\alpha}\right)}{2 a t^{\alpha}}\right]=u(z, t)\left[-\frac{\left(z-b t^{\alpha}\right)}{a t^{\alpha}}\right]
$$

After substitution of (3.7) into (3.6), the following relationship can be written down

$$
u(z, t)[\underbrace{-\frac{\left(z-b t^{\alpha}\right)}{a t^{\alpha}} \theta(z, t)}_{I-L}+\underbrace{\frac{\partial \theta(z, t)}{\partial z}}_{I I-L}]=u(z, t)[\underbrace{\frac{2\left(z-b t^{\alpha}\right) b \alpha t^{\alpha}+\left(z-b t^{\alpha}\right)^{2} \alpha}{2 a t^{\alpha+1}}}_{I-P} \underbrace{-\frac{\alpha}{2 t}}_{I I-P}]
$$

Using relationship (3.8), the function $\theta(z, t)$ is determined in such a way that the left side of relationship (3.8) equals its right side. Therefore

$$
\begin{aligned}
& I-L=I-P \Rightarrow \theta(z, t)=-\frac{2 b \alpha t^{\alpha}+\alpha\left(z-b t^{\alpha}\right)}{2 t} \\
& I I-L=I I-P \Rightarrow \frac{\partial \theta(z, t)}{\partial z}=-\frac{\alpha}{2 t}
\end{aligned}
$$

After reduction

$$
\theta(z, t)=-\frac{\alpha\left(z+b t^{\alpha}\right)}{2 t}
$$

The conclusion is that the primitive of the integrand takes the following form

$$
w(z, t)=u(z, t)\left[-\frac{\alpha\left(z+b t^{\alpha}\right)}{2 t}\right]
$$

where

$$
u(z, t)=\frac{1}{\sqrt{2 \pi a t^{\alpha}}} \exp \left(-\frac{\left(z-b t^{\alpha}\right)^{2}}{2 a t^{\alpha}}\right)
$$


We calculate integral (3.3)

$$
f(t)_{z_{d}}=u\left(z_{d}, t\right) \frac{\alpha\left(z_{d}+b t^{\alpha}\right)}{2 t}
$$

where

$$
u\left(z_{d}, t\right)=\frac{1}{\sqrt{2 \pi a t^{\alpha}}} \exp \left(-\frac{\left(z_{d}-b t^{\alpha}\right)^{2}}{2 a t^{\alpha}}\right)
$$

Thus, relationship (3.12) determines the density function of time of exceeding the boundary (permissible) condition by the diagnostic parameter $z_{d}$ deviation

$$
f(t)_{z_{d}}=\frac{\alpha\left(z_{d}+b t^{\alpha}\right)}{2 t} \frac{1}{\sqrt{2 \pi a t^{\alpha}}} \exp \left(-\frac{\left(z_{d}-b t^{\alpha}\right)^{2}}{2 a t^{\alpha}}\right)
$$

\section{A method to assess the lifetime of a device with respect to the diagnostic parameter being analyzed}

The formula for the reliability of a device with respect to the diagnostic parameter deviation from the nominal value can be expressed as

$$
R(\tau)=1-\int_{0}^{\tau} f\left(t, z_{d}\right) d t
$$

where

$$
f\left(t, z_{d}\right)=\frac{\alpha\left(z_{d}+b t^{\alpha}\right)}{2 t} \frac{1}{\sqrt{2 \pi a t^{\alpha}}} \exp \left(-\frac{\left(z_{d}-b t^{\alpha}\right)^{2}}{2 a t^{\alpha}}\right)
$$

Thus, the unreliability of a device can be expressed by the following formula

$$
Q(\tau)=\int_{0}^{\tau} \frac{\alpha\left(z_{d}+b t^{\alpha}\right)}{2 t} \frac{1}{\sqrt{2 \pi a t^{\alpha}}} \exp \left(-\frac{\left(z_{d}-b t^{\alpha}\right)^{2}}{2 a t^{\alpha}}\right) d t
$$

One can simplify integral (4.3) by making the following substitution $u=t^{\alpha}$

$$
Q(u)=\int_{0}^{\sqrt[\alpha]{u}} \frac{z_{d}+b u}{2 u} \frac{1}{\sqrt{2 \pi a u}} \exp \left(-\frac{\left(z_{d}-b u\right)^{2}}{2 a u}\right) d u
$$

Integral (4.4) should be converted into a simpler form and the problem comes down to solving the indefinite integral

$$
\int f\left(u, z_{d}\right) d u
$$

where $f\left(u, z_{d}\right)$ is the integrand of equation (4.5).

For the integrand of integral (4.5), the following change $\left(z_{d}-b u\right)^{2}=\left(b u-z_{d}\right)^{2}$ and substitution are performed $\omega=\left(b u-z_{d}\right)^{2} /(2 a u)$

$$
\frac{1}{2 \sqrt{\pi}} \int \frac{1}{\sqrt{\omega}} \mathrm{e}^{-\omega} d \omega
$$


The following substitution into integral (4.6), $\sqrt{\omega}=w$ results in what follows

$$
\frac{1}{2 \sqrt{\pi}} \int \frac{1}{\sqrt{\omega}} \mathrm{e}^{-\omega} d \omega=\frac{1}{\sqrt{\pi}} \int \mathrm{e}^{-w^{2}} d w
$$

With one more substitution into the integral (4.7)

$$
w^{2}=\frac{y^{2}}{2} \quad 2 w d w=y d y
$$

formula (4.7) can be written down as

$$
\frac{1}{\sqrt{\pi}} \int \mathrm{e}^{-w^{2}} d w=\frac{1}{\sqrt{2 \pi}} \int \exp \left(-\frac{y^{2}}{2}\right) d y
$$

Considering the above substitution $y$ takes the form expressed by

$$
y=\frac{\sqrt{2}\left(b t^{\alpha}-z_{d}\right)}{\sqrt{2 a t^{\alpha}}}
$$

Hence, remembering about the appropriate notation for the limits of integration, the unreliability of a device with respect to the growth of the diagnostic parameter deviation will be expressed by the following equation

$$
Q(\tau)=\frac{1}{\sqrt{2 \pi}} \int_{-\infty}^{\frac{\sqrt{2}\left(b t^{\alpha}-z_{d}\right)}{\sqrt{2 a t^{\alpha}}}} \exp \left(-\frac{y^{2}}{2}\right) d y
$$

Assuming some specific level of risk of failure, i.e. the level of probabilities of exceeding the permissible value of the parameter deviation, the following equation can be written down

$$
Q(\tau)=Q^{*}
$$

Hence

$$
Q^{*}=\frac{1}{\sqrt{2 \pi}} \int_{-\infty}^{\gamma} \exp \left(\frac{-y^{2}}{2}\right) d y
$$

For the assumed value of $Q^{*}$, the value of the upper limit of integral (4.13) is to be found in the Standard Normal Distribution Table.

In this way, we obtain the value of $\gamma$. Thus, the equation to determine lifetime of an element takes the following form

$$
\gamma=\frac{\sqrt{2}\left(b t^{\alpha}-z_{d}\right)}{\sqrt{2 a t^{\alpha}}}
$$

Having solved equation (4.14), one can find the lifetime of a device with respect to the assumed diagnostic parameter.

Therefore, having the value of the parameter $\bar{\gamma}$ and using relation (4.14), one can determine the lifetime of the device with respect to the diagnostic parameter given consideration. The following substitution has been made to do this

$$
s=t^{\alpha}
$$

Using formula (4.15), one can determine the lifetime of the device on the basis of the following relationship

$$
t^{*}=\frac{1}{a} \ln \frac{2 b z_{d}+\bar{\gamma}^{2} a+\bar{\gamma} \sqrt{4 b z_{d} a+\bar{\gamma}^{2} a^{2}}}{2 b^{2}}
$$




\section{Example of calculation}

To start verification of the method intended to determine the lifetime of a device with the Weibull distribution parameters applied, the coefficients of this distribution should be determined first. Both the parameter $\theta$ reflecting the scale factor and the parameter $\alpha$ representing the ratio of the shape have been pre-assumed to equal unity (determination thereof will provide the basis for further work).

Data recorded in the course of operating one of the sighting system units, i.e. the sighting head, has been assumed a good example, on the basis of which the above-presented model has been verified. The construction of this head enables visualisation of the sighting data in the form of the sighting marker that supports the sighting process while making use of air weapons. Under maintenance procedures/works performed every 100 hours' flight time recorded are values of diagnostic parameters of the sighting head in form of two co-ordinates $\varepsilon$ and $\beta$ that define the co-ordinates of the check position of the sighting marker. Nominal values of these co-ordinates (with permissible error range included) are determined by the aircraft manufacturer responsible for adjusting the sighting head with the sighting system at the stage the aircraft is introduced in service. If in the course of maintenance, the measured values of co-ordinates of the sighting marker position are within the limits of permissible error, no maintenance efforts are taken to correct the sighting marker position. On the other hand, if the value of at least one of the two diagnostic parameters exceeds the permissible error value, the sighting system is subjected to maintenance to remove the error. This is done by means of introducing into the system the co-ordinates that remove the deviation of the sighting marker position from the nominal one.

With data recorded in the course of the operational process $\left(\varepsilon_{1}, t_{1}\right) ;\left(\varepsilon_{2}, t_{2}\right) ; \ldots ;\left(\varepsilon_{n}, t_{n}\right)$ and related to changes in values of one of the parameters that define health/maintenance status of the sighting system, the rate of changes (of the increase) in deviation of the diagnostic parameter can be written down as

$$
c t_{1}=\varepsilon_{1} \quad c=\frac{\varepsilon_{1}}{t_{1}}
$$

For the data presented in Fig. 1, recorded in the course of maintenance works every 100 hours' flight time of the aircraft, the following parameters have been found that enable determination of lifetime described with relationship (4.14)

$$
b=0.00123 \quad a=1.524 \cdot 10^{-6} \quad c=0.00123
$$

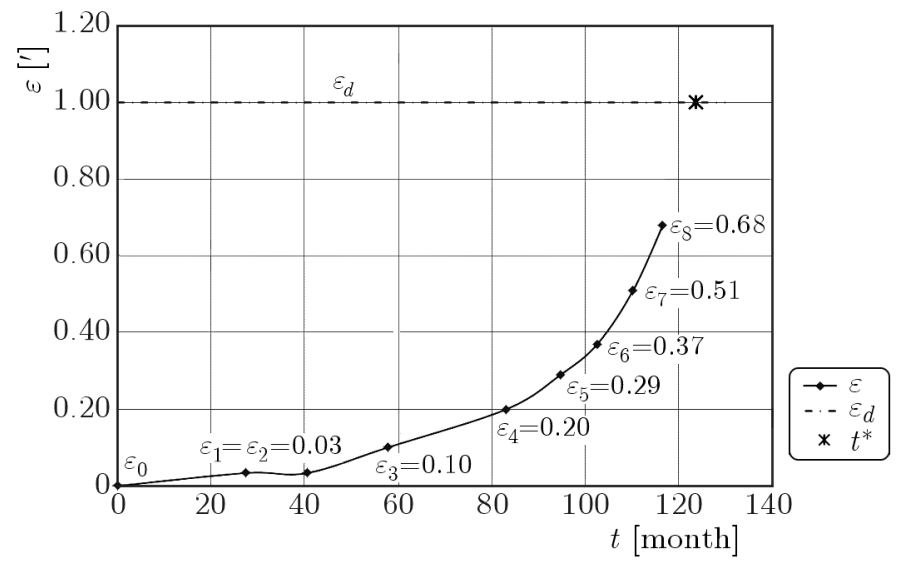

Fig. 1. Changes in the value of the diagnostic parameter that describes the position of the sighting marker and the value of the lifetime found

It is important for aeronautical systems that the level of reliability of the device in question nears unity. That is why the reliability has been assumed close to unity to determine the lifetime 
of the device. Assuming the level of reliability $R^{*}(t)=0.98$, the value of the parameter $\gamma^{*}=2.32$ could be found from the Standard Normal Distribution Tables. The parameter $\varepsilon_{d}$ could be found in the system technical documentation (Maintenance Manual), where information is given on the value of permissible deviation of the diagnostic parameter that describes co-ordinates of the sighting marker position. Using operational data (Fig. 1) and the above-determined parameters, the lifetime has been calculated from Eq. (4.14). In the analyzed case, it is

$$
t^{*}=124 \text { months }
$$

\section{Summary}

The process of operating technical devices installed on an aircraft involves influences of changeable weather and mechanical conditions resulting from, among other things, in-flight overloads. These factors lead to the accumulation of destructive factors affecting the system(s) and causing the nominal performance characteristics of selected system components decline. The material presented in this article is a continuation of analyses to develop methods of finding density function of changes in deviations of parameters, and the time of exceeding the permissible condition by the diagnostic parameter under analysis, the parameter being affected by particular destructive factors (Ważny, 2011a,b). The presented method enables determination of residual lifetime of a device and can be used to modify the process of operating the device in question. In this way, the number of checks of health/maintenance status can be limited, which consequently can reduce the time the device remains beyond the operational-use system.

The relationship for the failure rate described by parameters typical of the Weibull distribution, as applied to determine the rate of wear and tear of the component/device, is an important part of this study. Further work should be focused on verification of the method in question as referred to larger population of objects under analysis. Parameters that describe the rate of the ever increasing intensity of the component/device wear and tear should be found. Both objectives determine future lines of activity for the Author.

The above-presented considerations have been based on analyses of the process of operating aeronautical devices/systems. However, they can be successfully applied for other technical objects, the health/maintenance status of which is usually determined by means of diagnostic parameters. Implementation of this method is possible if we, firstly, have values of diagnostic parameters located along the axis of time of the device service/operation and, secondly, know the value of the boundary/limiting error that - when exceeded by the diagnostic parameter - makes operation of the device less effective (i.e. the device is at the medium level of serviceability).

\section{References}

1. Abezgauz G., 1973, Calculus Probabilistic. Handbook (in Polish), Wydawnictwo MON, Warszawa

2. Gniedenko B.W., Bielajew J.K., SoŁowiew A.D., 1968, Mathematical Methods in Reliability Theory (in Polish), WNT, Warszawa

3. Gradsztejn I.S., RyŻyk I.M., 1964, Tables of Integrals, Sums, Rows and Products (in Polish), PWN, Warszawa

4. Niu G., Singh S., Holland S., Pecht M., 2011, Health monitoring of electronic products based on Mahalanobis distance and Weibull decision metrics, Microelectronics Relibility, 51, 279-284

5. PamuŁa W., 2011, The Reliability and the Safety (in Polish), Wydawnictwo Politechniki Sląskiej, Gliwice 
6. Tomaszek H., Jasztal M., Zieja M., 2011, A simplified method to assess fatigue life of selected structural components of an aircraft for a variable load spectrum, Eksploatacja i Niezawodność Maintenance and Reliability, 4, 29-34

7. Tomaszek H., Jasztal M., Zieja M., 2013, Application of the Paris formula with $m=2$ and the variable load spectrum to a simplified method for evaluation of reliability and fatigue life demonstrated by aircraft components, Eksploatacja i Niezawodność - Maintenance and Reliability, 4, 297-304

8. Tomaszek H., Szczepanik R., 2005, Method of evaluation distribution of time of exceed a limiting state (in Polish), Zagadnienia Eksploatacji Maszyn, 40, 4, 35-44

9. Tomaszek H., Wróblewski M., 2001, Bases of Assessment of the Effectiveness of the Use of Weapon Systems Air (in Polish), Dom Wydawniczy Bellona, Warszawa

10. Tomaszek H., Żurek J., StęPIEń S., 2008, The airship maintenance with its renovation and the risk of its loss, Scientific Problems of Machines Operation and Maintenance, 43, 4, 39-49

11. Tomaszek H., Żurek J., Loroch L., 2004, The outline of a method of estimation reliability and durability of aircraft's structure elements on the basis of destruction process description (in Polish), Zagadnienia Eksploatacji Maszyn, 39, 3, 73-85

12. WAŻ́Y M., 2011a, An outline of the method for determining the density function of changes in diagnostic parameter deviations with the use of the Weibull distribution, Scientific Problems of Machines Operation and Maintenance, 46, 2, 105-144

13. WAŻNY M., 2011b, An outline of a method for determining the density function of the time of exceeding the limit state with the use of the Weibull distribution, Scientific Problems of Machines Operation and Maintenance, 46, 3, 77-91

14. WAŻny M., 2013, Effectiveness Analysis of the Air Sighting System in the Aspect of the Effect of Destructive Processes (in Polish), WAT

15. Ye Z., TANG L., XIE M., 2011, A burn-in scheme based on precentiles of the residual life, Journal of Quality Technology, 43, 4, 334-345

16. Zhanshan M., Krings, A.W., 2008, Survival analysis approach to reliability, survivability and prognostics and health management (PHM), Aerospace Conference, IEEE, 1-20 